\title{
Inter- and intra-breed genome-wide copy number diversity in a large cohort of European equine breeds
}

Marina Solé ${ }^{1 * \dagger} \mathbb{B}$, Michela Ablondi ${ }^{2 \dagger}$, Amrei Binzer-Panchal $^{3+}$, Brandon D. Velie ${ }^{4}$, Nina Hollfelder ${ }^{3}$, Nadine Buys $^{5}$, Bart J. Ducro ${ }^{6}$, Liesbeth François ${ }^{5}$, Steven Janssens ${ }^{5}$, Anouk Schurink ${ }^{6,7}$, Åsa Viklund ${ }^{1}$, Susanne Eriksson ${ }^{1}$, Anders Isaksson ${ }^{3}$, Hanna Kultima ${ }^{3}$, Sofia Mikko ${ }^{1+}$ and Gabriella Lindgren ${ }^{1,5+}$

\begin{abstract}
Background: Copy Number Variation (CNV) is a common form of genetic variation underlying animal evolution and phenotypic diversity across a wide range of species. In the mammalian genome, high frequency of CNV differentiation between breeds may be candidates for population-specific selection. However, CNV differentiation, selection and its population genetics have been poorly explored in horses.

Results: We investigated the patterns, population variation and gene annotation of CNV using the Axiom ${ }^{\circledast}$ Equine Genotyping Array $(670,796$ SNPs) from a large cohort of individuals $(N=1755)$ belonging to eight European horse breeds, varying from draught horses to several warmblood populations. After quality control, 152,640 SNP CNVs (individual markers), 18,800 segment CNVs (consecutive SNP CNVs of same gain/loss state or both) and 939 CNV regions (CNVRs; overlapping segment CNVs by at least $1 \mathrm{bp}$ ) compared to the average signal of the reference (Belgian draught horse) were identified. Our analyses showed that Equus caballus chromosome 12 (ECA12) was the most enriched in segment CNV gains and losses ( 3\% average proportion of the genome covered), but the highest number of segment CNVs were detected on ECA1 and ECA20 (regardless of size). The Friesian horses showed private SNP CNV gains (> 20\% of the samples) on ECA1 and Exmoor ponies displayed private SNP CNV losses on ECA25 (> 20\% of the samples). The Warmblood cluster showed private SNP CNV gains located in ECA9 and Draught cluster showed private SNP CNV losses located in ECA7. The length of the CNVRs ranged from $1 \mathrm{~kb}$ to 21.3 Mb. A total of 10,612 genes were annotated within the CNVRs. The PANTHER annotation of these genes showed significantly under- and overrepresented gene ontology biological terms related to cellular processes and immunity (Bonferroni $P$-value $<0.05$ ). We identified 80 CNVRs overlapping with known QTL for fertility, coat colour, conformation and temperament. We also report 67 novel CNVRs.
\end{abstract}

Conclusions: This work revealed that CNV patterns, in the genome of some European horse breeds, occurred in specific genomic regions. The results provide support to the hypothesis that high frequency private CNVs residing in genes may potentially be responsible for the diverse phenotypes seen between horse breeds.

Keywords: Copy number variation, Horse, Structural variation, SNP genotyping array

\footnotetext{
* Correspondence: marina.sole@slu.se

${ }^{+}$Marina Solé, Michela Ablondi and Amrei Binzer-Panchal shared authorship.

†Sofia Mikko and Gabriella Lindgren shared senior authorship.

${ }^{1}$ Department of Animal Breeding \& Genetics, Swedish University of

Agricultural Sciences, Uppsala, Sweden

Full list of author information is available at the end of the article
}

(c) The Author(s). 2019 Open Access This article is distributed under the terms of the Creative Commons Attribution 4.0 International License (http://creativecommons.org/licenses/by/4.0/), which permits unrestricted use, distribution, and reproduction in any medium, provided you give appropriate credit to the original author(s) and the source, provide a link to the Creative Commons license, and indicate if changes were made. The Creative Commons Public Domain Dedication waiver (http://creativecommons.org/publicdomain/zero/1.0/) applies to the data made available in this article, unless otherwise stated. 


\section{Background}

With the development of genome-wide genotyping arrays, genetic diversity and the variation of complex phenotypes are often assessed via single nucleotide polymorphism (SNP). However, variation in SNPs only explains a fraction of the genetic component of phenotypic variance. Recently, increased attention has been drawn to copy number variations (CNVs), as they are widely considered to impact phenotypes. CNVs are typically defined as variation due to deletions, insertions, and duplication events (1 kilobase-pairs $(\mathrm{kb})$ to several megabase-pairs $(\mathrm{Mb})$ ) when comparing the genomic sequence of individuals with a reference genome [1].

The total number of CNVs could explain much of the heritability unaccounted for by SNPs as CNVs have been linked to genetic variation of complex traits influencing phenotypic diversity and disorders across a wide range of species [2-11]. In horses, it has been shown that $\mathrm{CNVs}$ can cause phenotypic variation and disorders. A 4.6-kb intronic duplication in STX17 on ECA25 has been linked to hair greying and melanoma [12], and large deletions at the $S H O X$ locus in a pseudoautosomal region are associated with Skeletal Atavism in Shetland Ponies [13]. Furthermore, studies in dogs have been shown that between 3 and 24\% of unique CNVs potentially contribute to phenotypic diversity [9]. For instance, the breed characteristic dorsal hair in Rhodesian and Thai Ridgebacks has been linked to a duplication of a set of FGF genes, which also predispose to dermoid sinus disorder [11].

There are also some mammalian gene super-families commonly known to underpin evolutionary changes driven by CNVs, where genes associated to $\mathrm{CNV}$ gains may be a likely instrument of adaptation [14]. Consequently, most of the variation explained by CNVs in the mammalians' genomes is known to occur in regions that regulate important biological processes such as sensory perception, signal transduction, immunity and pathogen defence or metabolism pathways [2, 5, 7-9, 14]. Therefore, the analysis of $\mathrm{CNVs}$ in domestic and livestock species has become increasingly important for the evaluation of genetic diversity, phenotypic variation and complex phenotypes.

The current publicly available database of genetic variants in the equine genome contains 25,756,212 SNP and $3,663,455$ insertion/deletions polymorphisms "INDELs", accessed from the Genome Variation Map (http://bigd. big.ac.cn/gvm/home). Until now, CNVs identified in nearly 45 different horse breeds occupy about $1-3 \%$ of their genome and there are more $\mathrm{CNVs}$ residing in genes $(\sim 80 \%)$ than in intergenic regions $(\sim 20 \%)$ [5, 7, 15-19]. In horses, the average range for $\mathrm{CNV}$ size remains between $1 \mathrm{~kb}$ to $4.84 \mathrm{Mb}$ with CNV losses generally dominating over gains in comparison with a reference Thoroughbred genome [7, 15-17]. The majority of the actual number of identified specific CNVs in horses has been reported using a limited number of individuals [7, 17, 20]. Although significant associations with specific traits such as body size or recurrent laryngeal neuropathy have been detected using large sample datasets $[18,19]$.

Previous studies in other mammals have hypothesized that CNVs with high frequency differences among breeds are involved in population-specific selection and adaptation to the environment [21-23], and also that CNVs residing in genes contribute more to population differentiation and divergence [24]. However, CNV differentiation, selection and its population genetics have been poorly explored in horses. As such, in the present study we aimed to 1 ) investigate the distribution pattern of CNVs, 2) detect breed specific CNVs and 3) identify biological pathways affected by CNVs in the horse genome using a large cohort of individuals and high density (HD)-SNP genotyping array data across eight European equine breed populations (Ardenner, Belgian draught, German draught, Exmoor, Vlaams paard, Friesian horses, Belgian Warmblood and Swedish Warmblood).

\section{Results \\ Copy number variations in various European horse populations}

Genome-wide CNV analysis was conducted on 1755 horses from 8 different breeds (Table 1) using the Affymetrix Axiom ${ }^{\text {sm }}$ Equine HD-SNP genotyping array (MNEC670K) [25]. CNVs were analysed on autosomes by comparing the ratio of signal intensities of each sample with the average signal of the reference (Belgian draught horse; extensive sample size $(N=301)$ and the lowest variation in signal intensity among the studied breeds). After quality control, we identified 152,640 SNP CNVs (signal gains or losses specific to each individual and state), 18,800 segment CNVs (consecutive SNP CNVs per individual of same gain/loss state or both), and $939 \mathrm{CNV}$ regions (CNVRs; overlapping segment CNVs by at least $1 \mathrm{bp}$ in the whole population per state: signal gain, loss or both).

To shed light on the diversity between breeds and their CNVs distribution, we used principal component analysis (PCA). First, the plot for the SNP B-allele frequency ratios (normalized measure of the allelic intensity ratio of the two alleles) showed a clear separation of the individuals in three main breed groups on PC2, with the Friesian horses uniquely distinguished from the other horse breed populations, that clustered in two main subpopulations; i) Warmblood horses, and ii) Draught horses including Exmoor ponies (Fig. 1a).

Interestingly, the first principal component represented variation between individuals, across breeds (PC1 explained $61.9 \%$ of the total variance), and the second principal component represented variation between 
Table 1 Descriptive statistics for Copy Number Variations (segment CNVs)

\begin{tabular}{|c|c|c|c|c|c|c|c|}
\hline Breeds & $\mathrm{N}$ of individuals & $\%$ total change & sd total change & $\%$ gain & sd gain & $\%$ loss & sd loss \\
\hline \multicolumn{8}{|l|}{ Draught } \\
\hline Ardenner & 24 & 0.2 & 0.05 & 0.1 & 0.05 & 0.1 & 0.04 \\
\hline Belgian draught ${ }^{\mathrm{a}}$ & 301 & 0.2 & 0.23 & 0.1 & 0.07 & 0.1 & 0.22 \\
\hline German draught & 22 & 0.2 & 0.09 & 0.1 & 0.08 & 0.1 & 0.04 \\
\hline Exmoor ponies & 256 & 0.2 & 0.43 & 0.2 & 0.41 & 0.1 & 0.10 \\
\hline Vlaams paard & 22 & 0.2 & 0.08 & 0.2 & 0.07 & 0.1 & 0.03 \\
\hline \multicolumn{8}{|l|}{ Warmblood } \\
\hline Belgian Warmblood I & 234 & 0.3 & 0.11 & 0.2 & 0.12 & 0.1 & 0.04 \\
\hline Belgian Warmblood II & 247 & 0.4 & 0.92 & 0.3 & 0.84 & 0.1 & 0.09 \\
\hline Swedish Warmblood & 383 & 0.3 & 0.21 & 0.2 & 0.19 & 0.1 & 0.08 \\
\hline \multicolumn{8}{|l|}{ Friesian } \\
\hline Friesian horses & 266 & 0.9 & 1.10 & 0.7 & 1.10 & 0.1 & 0.12 \\
\hline
\end{tabular}

Average and standard deviation (sd) of the proportion of the total genome (without sex chromosomes) with a percent total change of segment CNVs, percentage gains of segment CNVs, and percent losses of segment CNVs, compared to the average signal of the reference ${ }^{\mathrm{a}}$

breeds (PC2 explained $3.1 \%$ of the total variance). In contrast, PCAs generated from segment CNVs showed less variation across breeds (Fig. 1b). However, Friesian horses were the most differentiated with $0.9 \%$ of total segment $\mathrm{CNV}$ changes in their genome when compared to the reference (Table 1).

The distribution of segment CNVs showed several overlapping regions in the genome of the studied breeds. The largest percentage of the genome covered by segment $\mathrm{CNV}$ gains and losses $(\sim 2.5-3.0 \%$ average percentage of the genome covered; Fig. 2) was detected in ECA12, but the highest percentages of number of segment CNVs (above 10\%) were detected on ECA1 and ECA20 (regardless of the chromosomal genome size; Fig. 3).

\section{Private CNV comparisons}

The number of locations with unique private $\mathrm{CNV}$ gains and losses relative to the reference breed varied between the populations. In total, the number of genome positions that displayed SNP CNV gains ranged between 4629 (Ardenner) and 56,033 (Friesian horses) (Table 2). The average number of SNP CNV gains per individual ranged between 25.14 (Belgian draught horse) and 332.64 (Ardenner). The number of genome positions observed with SNP CNV losses ranged between 3715 (Vlaams paard) and 7142 (Friesian horses) (Table 2), whereas the average number of SNP CNV losses per individual ranged between 13.22 (Swedish Warmblood) and 168.86 (Vlaams paard). In this sense, percentage of unique private SNP CNV gains in relation to the total number of gains within breed ranged between $0.1 \%$ (Belgian warmblood I) and 10.5\% (Friesian horses), and percentage of unique private SNP CNV losses in relation to the total number of losses ranged between $0.4 \%$ (Ardenner) and 14.0\% (Exmoor ponies). The within population percentage, and positions of the unique private SNP CNVs are plotted in Fig. 4. The Friesian horses showed the largest percentage of unique private SNP CNV gains ( $>20 \%$ of the samples) on ECA1 and Exmoor ponies displayed the largest percentage of unique private SNP CNV losses on ECA25 (> 20\% of the samples).

\section{Breed cluster CNV comparisons}

To better understand patterns of CNVs between breeds of common ancestral origin, we grouped the breeds in three breed clusters according to the previous PCA analysis (Fig. 1a; Draught including Exmoor ponies, Warmblood and Friesian horses [26]). The total number of genomic locations presenting SNP CNVs (signal gains and losses) and the percentage of unique private SNP $\mathrm{CNV}$ gains or losses corresponding to the breed group clusters are shown in Table 3. The Friesian horses showed the largest average number of SNP CNV gains and losses per individual (210.65 and 26.85, respectively). The percentage of unique private SNP CNV gains in relation to the total number of gains within breed group was higher in the Friesian horses (10.5\%), and the percentage of unique private SNP CNV losses in relation to the total number of losses was similar in the Draughts and Warmbloods (18.7 and 19.2\%, respectively).

The chromosomal percentage distribution of unique private SNP CNV gains and losses across the entire genome of the three breed clusters is shown in Fig. 5. Particularly, the Friesian horses showed a high percentage of unique private SNP CNVs gains (> $20 \%$ of the samples) at the same location shown in previous plot (ECA1, Fig. 3). The Warmbloods showed genomic regions with high percentage ( $>20 \%$ of the samples) of unique private SNP CNV gains in ECA9 (Fig. 5) compared to the reference. The Draughts also showed 

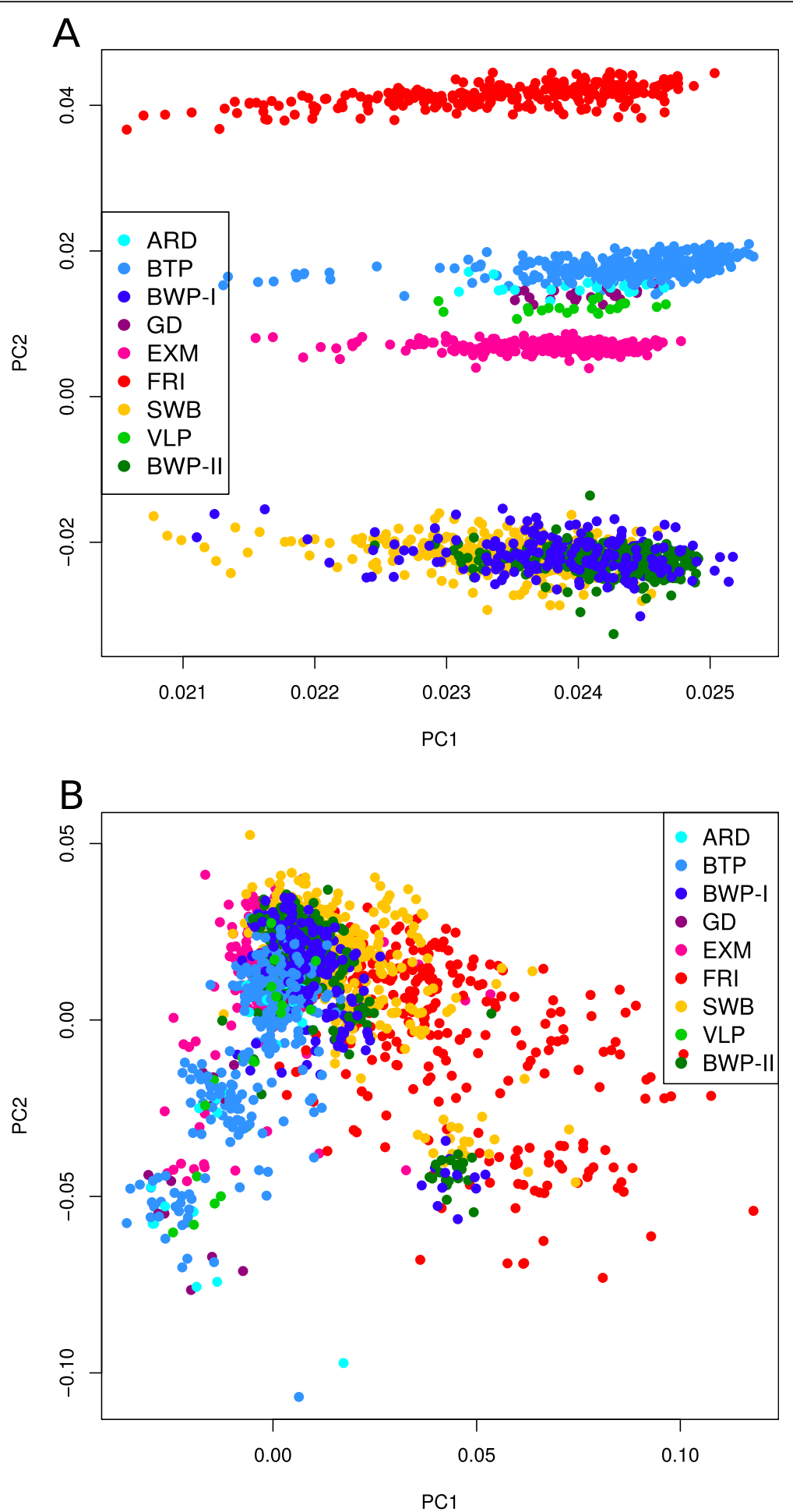

Fig. 1 Principal component analysis across the eight European horse breeds. a (left) SNP B-allele frequency ratios of the samples. $\mathbf{b}$ (right) CNV of the segments detected, compared to the average signal of the reference. ARD $=$ Ardenner; BTP $=$ Belgian draught horse ( ${ }^{*}$ reference); BWP-I and BWP- $\|=B e l g i a n$ Warmblood I and II; GD = German draught; EXM = Exmoor ponies; FRI = Friesian horses; SWB = Swedish Warmblood; VLP = Vlaams paard

regions with high percentage ( $>20 \%$ of the samples) of unique private SNP CNV losses in ECA7 (Fig. 5) compared to the reference.
CNV regions, gene annotation and PANTHER classification In the present study, we identified 18,800 segment CNVs representing 13,178 segment $\mathrm{CNV}$ gains (average length 


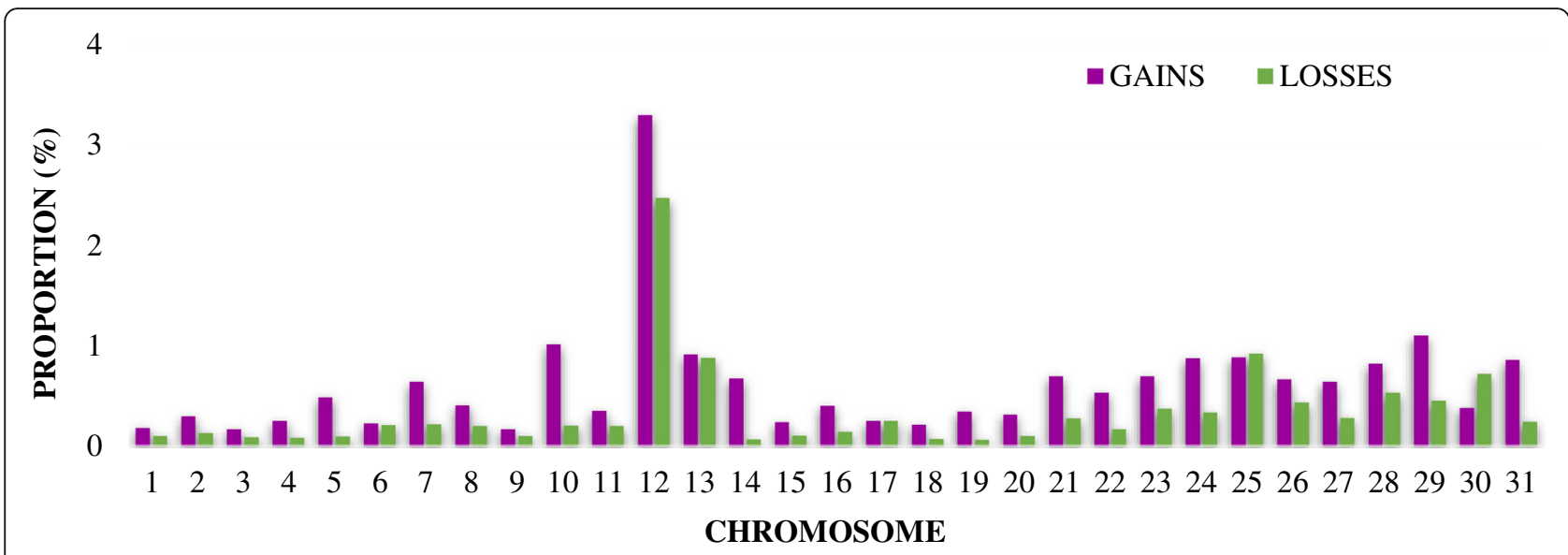

Fig. 2 Chromosomal distribution of the detected segment CNVs. Length of colored bars corresponds to the average proportion of the genome covered in percentage. Segment CNVs gains (purple), segment CNVs losses (green), compared to the average signal of the reference

of $414.78 \mathrm{~kb}), 5298$ segment CNV losses (average length of $196.95 \mathrm{~kb}$ ) and 324 segment $\mathrm{CNV}$ gains/losses (average length of $241.84 \mathrm{~kb}$ ), compared to the reference. Overlapping segment CNVs by at least $1 \mathrm{bp}$ were merged into 939 CNVRs (ranging from $1 \mathrm{~kb}$ to $21.3 \mathrm{Mb}$ length; Additional file 1: Table S1). The majority of CNVRs contained genes (79.3\%). Specifically, 10,612 genes were annotated within the CNVRs (Additional file 2). The PANTHER analysis showed significantly underrepresented gene ontology (GO) terms involved in single-stranded RNA binding, transposition, cellular response to lipopolysaccharide and ribonucleoprotein complex functions (Bonferroni $P$-value $<0.05$; Additional file 3: Table S2). Overrepresented GO terms involved in G-protein coupled receptor activity, immune response, immunoglobulin and membrane-bound signalling molecule functions (Bonferroni P-value <0.05; Additional file 3: Table S2). Additionally, we identified 80 CNVRs (ranging from $129 \mathrm{~kb}$ to $9.04 \mathrm{Mb}$ length) which overlapped with known QTLs for conformation, coat colour, fertility, hair and temperament traits (Additional file 1: Table S1).

\section{Novel CNVR and validation by RT-qPCR}

The majority of the identified CNVRs overlapped with at least $1 \mathrm{bp}$ with the previously identified and published equine CNVRs, while 67 out of 939 CNVRs were novel (7.1\%, (see Methods and Additional file 1: Table S1). To further verify the accuracy of CNVR prediction, RTqPCR was used to validate four genomic regions containing common segment CNVs of different sizes detected in this study (from $12 \mathrm{~kb}$ to $2 \mathrm{Mb}$ length). The CNVR located within Olfr4F21 gene on ECA1 and OR8S1 gene on ECA6 were already detected in previous studies $[15,20]$, whereas the remaining two were novel CNVRs. The CNVRs represented a different state of the segment CNVs (signal gain and loss in relation to the

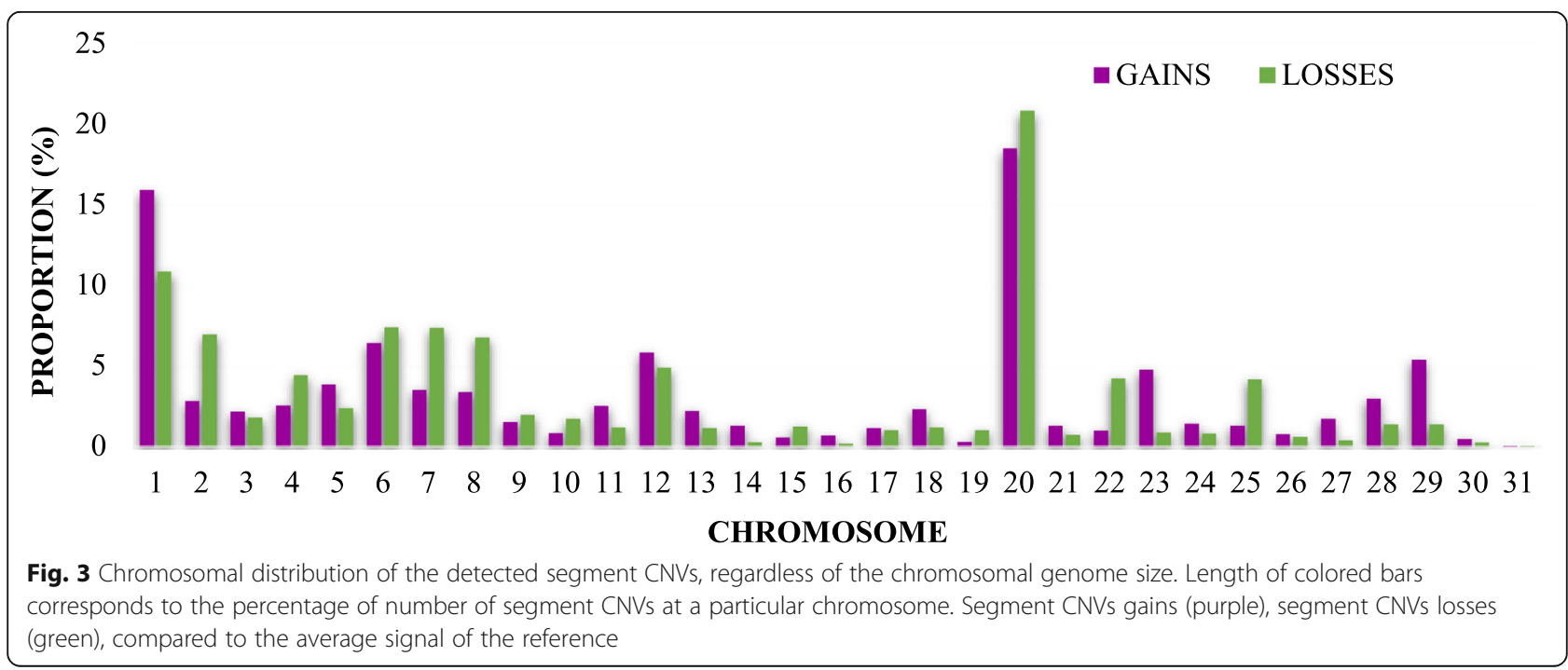


Table 2 Breed total number of genome positions that display SNP copy number gains and losses

\begin{tabular}{|c|c|c|c|c|}
\hline \multirow[t]{2}{*}{ Breeds } & \multicolumn{2}{|l|}{ SNP CNV } & \multicolumn{2}{|c|}{$\%$ Unique private $C N V^{b}$} \\
\hline & gains & losses & gains & losses \\
\hline \multicolumn{5}{|l|}{ Draught } \\
\hline Ardenner & 4629 (192.88) & $4324(180.17)$ & 3.1 & 0.4 \\
\hline Belgian draught horse $^{a}$ & $7568(25.14)$ & $5894(19.58)$ & 2.8 & 2.5 \\
\hline German draught & 5777 (262.59) & $3894(177.00)$ & 2.5 & 0.6 \\
\hline Exmoor ponies & $18,150(70.90)$ & $4236(16.55)$ & 6.5 & 14.0 \\
\hline Vlaams paard & 7318 (332.64) & 3715 (168.86) & 2.9 & 1.2 \\
\hline \multicolumn{5}{|l|}{ Warmblood } \\
\hline Belgian Warmblood I & $9837(42.04)$ & $5760(24.62)$ & 0.1 & 1.5 \\
\hline Belgian Warmblood II & $10,852(43.94)$ & $5473(22.16)$ & 2.4 & 1.6 \\
\hline Swedish Warmblood & $13,994(36.54)$ & $5064(13.22)$ & 0.9 & 1.1 \\
\hline \multicolumn{5}{|l|}{ Friesian } \\
\hline Friesian horses & $56,033(210.65)$ & $7142(26.85)$ & 10.5 & 1.4 \\
\hline
\end{tabular}

SNP CNVs compared to the average signal of the reference ${ }^{\text {a }}$. The average number of SNP CNVs / individual is given within brackets. The percentage of breed specific (unique private) SNP CNV gains and losses in the genome are also shown. ${ }^{\mathrm{b}}$ The sex chromosomes are not included in the total genome size. Every genome position showed a particular SNP CNV in at least $1 \%$ of the samples within breed

average signal of the reference; Additional file 4: Table S3). From the 80 sample-locus combinations tested, all four CNVRs were confirmed by RT-qPCR, as different copy number states were found per each CNVR.

Genotyping concordance rate between the Affymetrix Axiom array data and the RT-qPCR validation differed slightly between regions (Additional file 4: Table S4). In the case of the CNVR within the Olfr4F21 gene, the concordance rate was equal to $90 \%$ with one duplication in one horse not confirmed by RT-qPCR. Both the CNVRs located within OR10G2 and OR08S1 genes had a concordance rate of $80 \%$. For the CNVR located within the OR08S1 gene, two individuals showed homozygous one copy deletion by RT-qPCR which were not detected by the HD-SNP genotyping array. One deletion was not confirmed by RT-qPCR in the case of OR10G2 gene and one duplication was found by RTqPCR which was not previously detected from the SNP array. In the case of the CNVR located in the $S V 2 C$ gene, two samples showed homozygous one copy deletion in the RT-qPCR which were not detected from the HD-SNP genotyping array.

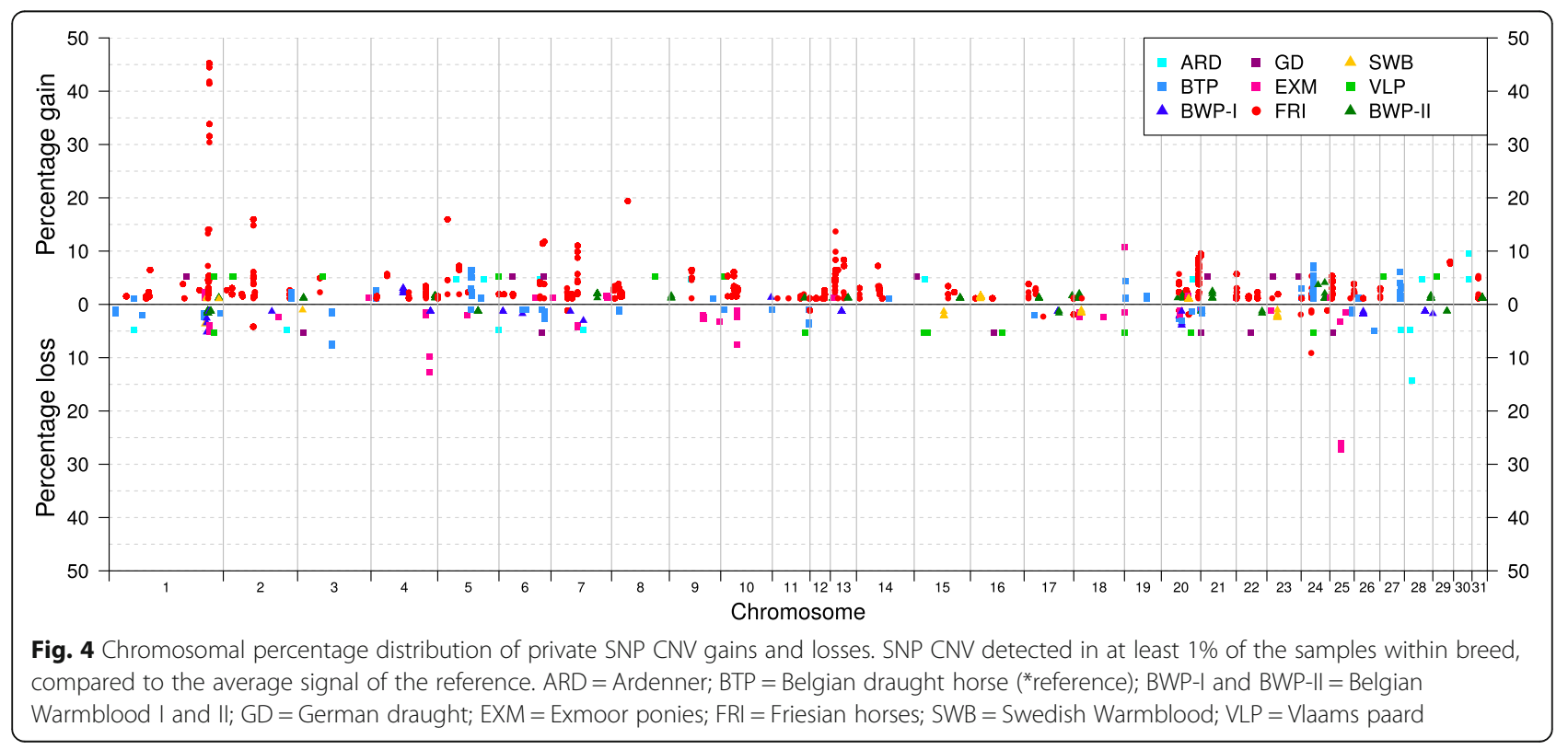


Table 3 Breed group total number of genome positions that display SNP copy number gains and losses

\begin{tabular}{|c|c|c|c|c|c|}
\hline \multirow[t]{2}{*}{ Breeds } & \multirow{2}{*}{$\begin{array}{l}\mathrm{N} \text { of } \\
\text { individuals }\end{array}$} & \multicolumn{2}{|l|}{ SNP CNV } & \multicolumn{2}{|c|}{$\%$ Unique private $\mathrm{CNV}^{\mathrm{a}}$} \\
\hline & & gains & losses & gains & losses \\
\hline Draught & 624 & $7527(12.06)$ & $6041(9.68)$ & 1.99 & 18.66 \\
\hline Warmblood & 864 & $13,133(15.20)$ & $5591(6.47)$ & 8.62 & 19.19 \\
\hline Friesian & 266 & $56,033(210.65)$ & $7142(26.85)$ & 10.47 & 1.43 \\
\hline
\end{tabular}

SNP CNVs compared to the average signal of the reference (Belgian draught horse). The average number of SNP CNVs / group cluster is given within brackets.

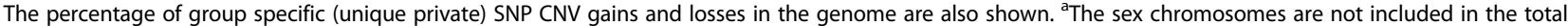
genome size. Every genome position showed a particular SNP CNV in at least $1 \%$ of the samples within breed group

\section{Discussion}

\section{Diversity of CNVs between different European horse breeds}

Genetic polymorphisms play an important role in the phenotypic diversification and speciation in equids [27, 28]. Although diverse genetic variants underlying phenotypic variation have been successfully mapped (e.g. [12, 13, 29, 30]), a large proportion of the horse genome still remains poorly understood. Using HD-SNP array data from a large cohort of individuals across groups of phenotypically and ancestrally divergent horses, we showed that $\mathrm{CNV}$ distribution across different breeds presented many commonalities (genomic location, gain or loss), but that some unique private $\mathrm{CNVs}$ were observed in particular genomic regions. Moreover, both validation rate of CNVRs and overall genotyping concordance rate of $82.5 \%$ proved the Axiom Analysis as a consistent method for CNV calling.

Principal component analysis showed remarkable variation among populations, and was in accordance with known breed divergence and history [26, 31]. Comparable findings were also pointed out in other domestic and livestock species [23, 32, 33]. Similar to the current study, previous studies have indicated clear distinctions in $\mathrm{CNV}$ frequency between breed groups or populations, possibly reflecting breed patterns of phenotypic diversity and the population history of different breeds, such as a change in past effective population size, gene flow, or selection $[9,21,23]$. Our observed differences also support the hypothesis that genetic variation from CNVs may contribute to breed phenotypic diversity, but it may also result from the differential demographic history and effective population sizes between breeds [9, 23].

With only minor exceptions, the CNV distribution, showed small differences in SNP and segment CNVs between breeds. The average percentage of total coverage by the segment CNVs identified across the genome of the investigated breeds was small in relation to the reference breed $(<0.5 \%)$. In general, low CNV diversity is expected [7] as the segment CNVs identified in the present study covered an even smaller proportion of the genome in comparison with those previously reported in the same type of horse breeds [5, 7, 16, 17]. Consequently, the differences observed may be attributable to the different genetic background of the individuals, sample size and methodologies applied for CNV discovery. As observed in other studies with the same type of horse breeds, the largest number of shared CNVRs were found in ECA12 (e.g. ECA12:9,158,392-17,707,943 [5, 7, 16, 18, 20]).

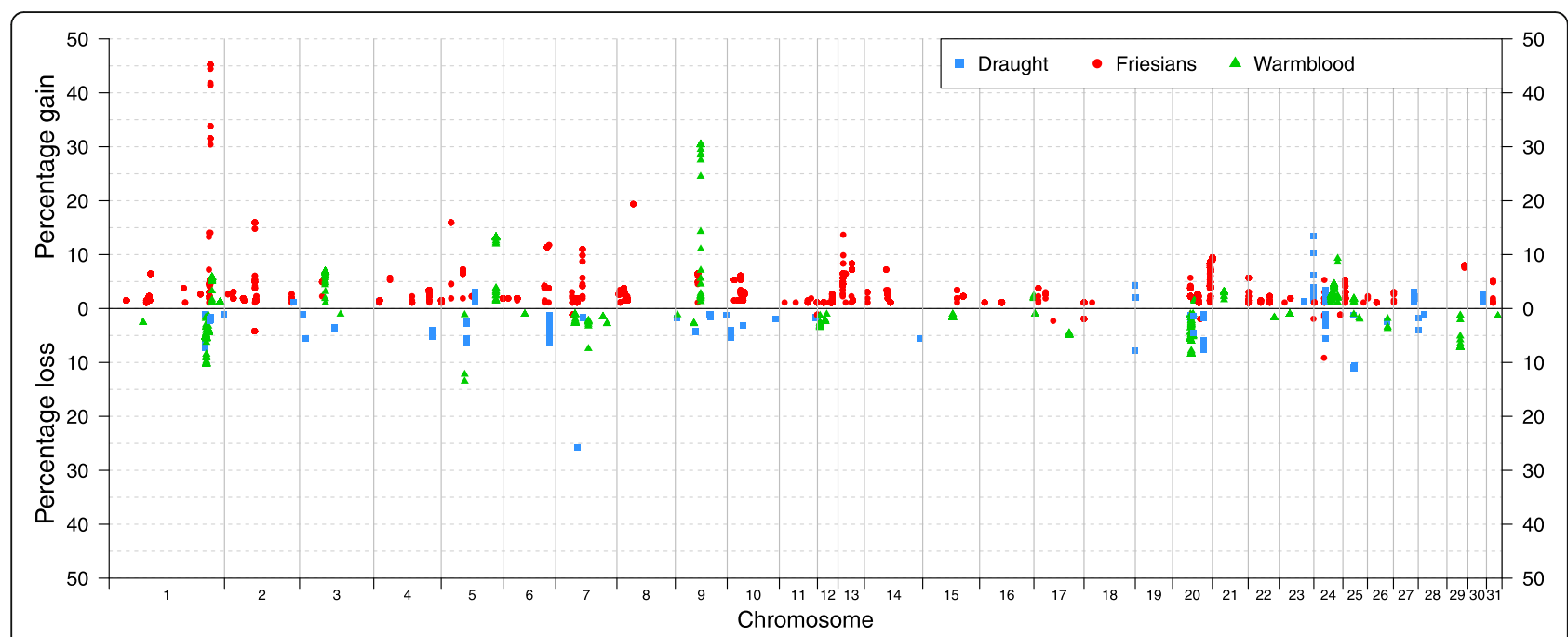

Fig. 5 Chromosomal percentage distribution of breed cluster-specific SNP CNV gains and losses. SNP CNV detected in at least 1\% of the samples within breed clusters, compared to the average signal of the reference 
ECA12 displays the particular feature of being enriched with clusters of olfactory receptor genes, which is also observed in other mammalian genomes and it has been hypothesized to influence flight response and temperament diversity in horses [7]. Similarly, we found overlapping of CNVRs with previously identified T-cell receptor and MHC class genes, that exhibited high levels of diversity in one or more similar type of horse breeds (e.g. on ECA1: 154,857,175-156,876,500; ECA1:158,843,180-160,751,024; and ECA20:28,731,700-35,604,382) $[5,7,16,18]$. Interestingly, ECA1 overlapped with established QTLs for conformation in British ponies and reproductive traits in German Warmbloods (e.g. on ECA1:53,917,112-54,139,087 and ECA1:93,506,091-95,186,154 [34, 35]), and CNVRs in ECA20 overlapped with QTLs for back conformation in American Saddlebred horses (e.g. on ECA20:41,994,712-43, 192,412 and ECA20:43,299,741-43,568,400 [36]). The largest amount of shared CNVRs within all horse breeds studied overlapped with QTLs for white markings detected in the light draught Franches-Montagnes horses (ECA1:154, 857,175-156,876,500 [37]) and withers at height detected in British ponies (ECA7:43,0483,386-53,094,544; ECA8:0-6, 769,072 and ECA12:9,158,392-17,707,943 [34]). Although no candidate genes have been previously reported in these regions, our findings suggest that the functionality of $\mathrm{CNV}$ enriched genes in horses fall into sensory perception, immunity, reproduction and exterior traits.

Our results also support the hypothesis that high frequency private SNP CNVs in particular (e.g. on ECA25 in Exmoor ponies) may be responsible for populationspecific selection and adaptation [21, 22]. This provides further evidence to presume that CNVs in these regions may represent a substantial source of genetic variation for diverse phenotypes and biological processes, although further analysis is required to confirm phenotypic changes. Additionally, a greater amount of segment $\mathrm{CNV}$ gains compared to losses was observed, potentially reflecting the large number of losses in the reference native Belgian draught horse. However, this may also reflect the fact that duplications of coding sequences potentially enhance the organisms' genetic diversity, phenotypic variation and adaptation potential $[38,39]$.

\section{Diversity of CNVs in breed clusters}

The large cohort of individuals analysed in this study $(\sim 195$ horse on average per breed), in comparison with other studies where breeds are represented with one or two horses, is likely to provide a more accurate overview of single private CNVs. The PCA analysis of the SNP and segment CNV distribution according to the three breed clusters confirmed similar frequency levels within three breed clusters. This should not be surprising given that the largest number of shared CNVs was previously reported between closely related breeds such as Warmbloods [18].
Our results also showed that the proportion of breed cluster-specific SNP CNVs differed between groups. For instance, the Draught and Warmblood clusters displayed a relatively high proportion of unique private SNP CNVs (up to $30-50 \%$ ). However, a smaller proportion of SNP CNVs may be attributable to breed-specific characteristics (less than 14\%). Such differences in unique CNVs in ancestrally divergent Equidae members have also been previously reported. As an example, Doan et al. [7] detected higher proportions of certain specific CNVs in donkeys (35\%) and miniature (24\%) horses, over the total breed-specific CNVs across 17 different Equidae species, which has been attributed to larger divergences relative to the Thoroughbreds.

Interestingly, we also identified breed group-specific SNP CNVs located in particular genomic regions which may be attributable to breed-group features. For instance, specific SNP CNVs gains were detected in ECA1 $(163,032,489-163,181,822)$ in more than $20 \%$ of the individuals belonging to the Friesian horses' cluster, and more than $20 \%$ of the individuals belonging to the Draught cluster showed specific SNP CNV losses in ECA7 (34,601,429-34,608,700). However, all these private SNP CNVs identified reside in intergenic regions or merge into CNVR which partially overlap with previously identified regions in the Quarter horse [17]. Notwithstanding, the identified SNP CNVs gains in ECA9 $(40,822,784-40,867,675)$ in more than $20 \%$ of the individuals belonging to the Warmblood cluster reside in a region which contain two genes, RAD54 homolog B (RAD54B) and Reactive intermediate imine deaminase A (RIDA). The latest is regarded as a potential candidate gene for athletic performance since it is involved in metabolic processes and has been related to blood protein levels in humans [40]. CNV polymorphisms in these region may represent a substantial source of genetic variation of high value for genetic association analyses in the future.

\section{PANTHER analysis of genes underlying the CNVR and novel CNVR discovery}

The evolutionary process of species formation (speciation) is complex and influenced by fast evolving changes in specific regions in the genome (i.e. CNVRs driving novel gene functions), which may affect regulatory key biological mechanisms and play a fundamental role in gradual adaptation to different environments $[1,22]$. In the last 10 years, substantial progress has been made in relation to the functional impact of structural variants in several species with focus on population diversity. Enrichment of CNVRs in genes related to immune response, brain development, metabolic processes, sensory perception of smell or chemical stimuli have been reported in global populations of humans as well as pigs, dogs, cattle 
and horses [2, 4, 8, 18, 23, 41]. In this sense, our results also indicate that CNVRs are located in specific genomic regions and are involved in important biological processes in mammals such as immunity. As horse populations have gone through strong and diverse selection since horse domestication [28], these findings could be expected and may indicate favourable selection of structural variants associated with specific traits (e.g. insect bite hypersensitivity $(\mathrm{IBH})$ in the Friesian horses [20]). However, the association of CNVs with certain private traits in horses needs further exploration.

\section{Conclusions}

Our PCA analysis of SNP and segment CNVs showed that horse populations tend to group according to breed ancestral origins. Comparing breed and breed group clusters, we identified potentially unique private genomic regions displaying SNP CNVs. We found small percentages (less than 14\%) of unique private intra-breed structural variants that may contribute to the equine breed diversity. Specifically, ECA9 displayed a high frequency of specific SNP CNVs gains in the Warmblood cluster in a region containing RIDA gene, involved in metabolic processes. Genes located within CNVRs demonstrated under- and overrepresentation of gene ontology biological terms related to cellular processes and immunity, suggesting the potential role of structural variants in driving phenotypic diversity and disease resistance in European horse populations. We identified 80 CNVRs overlapping with established fertility, coat colour, conformation and temperament trait QTLs. We also report 67 novel CNVRs, which contribute to the catalogue of known CNVs in the horse genome. Future research is needed in order to confirm if the observed CNVs across breeds are also linked to phenotypical differences.

\section{Methods}

Horses, breeds

The studied cohort comprised 1755 horses representing eight breeds (Table 1). The Friesian horse samples belonged to a half-sib case-control set-up to study IBH [20]. The Exmoor ponies had previously been genotyped for a case-control genome scan to detect genetic risk factors for IBH [42]. The Swedish Warmblood horses were selected randomly from four different performance groups representative of the SWB population in general. The rest of the horse breeds belonged to case-control set-ups for different diseases under the EU HORSEGENE project. Belgian Warmbloods were treated initially as 2 cohorts (BWP-I and BWP-II) as they differed in average birth year by 9 years (approx. 1 generation). Based on clustering results, all Warmbloods were merged together.

\section{DNA isolation}

Genomic DNA from the Friesian horses and Exmoor ponies was isolated as described in Schurink et al. [20] and Velie et al. [42]. DNA for Swedish Warmbloods was prepared from 20 hair roots, cut into 5\% Chelex 100 Resin (Bio-Rad Laboratories, Hercules, CA, US), and $1.4 \mathrm{mg} / \mathrm{ml}$ Proteinase K (Merck KgaA, Darmstadt, Germany) in a total volume of $200 \mu \mathrm{l}$. The samples were incubated at $56^{\circ} \mathrm{C}$, $1500 \mathrm{rpm}$ for $2 \mathrm{~h}$, followed by heat inactivation of Proteinase $\mathrm{K}$ at $96^{\circ} \mathrm{C}$ for $10 \mathrm{~min}$. DNA concentration was normalized, and the DNA was re-suspended in lowTE (1 mM Tris, $0.1 \mathrm{mM}$ EDTA) at a concentration of $10 \mathrm{ng} / \mu \mathrm{l}$. The QIAamp DNA Blood Midi Kit was used to isolate DNA from whole blood of the Belgian Warmblood, Belgian Draught, German Draught, Vlaams paard and Ardenner horses. Lower limits for genotyping where a concentration of at least $20 \mathrm{ng} / \mu \mathrm{l}$, a total amount of $300 \mathrm{ng}$ DNA per sample and an OD ratio 260/280 between 1.8-2.0 was used.

\section{Microarray analysis}

Genotyping was performed according to the standard protocol Axiom $^{\mathrm{mm}}$ 2.0 Assay Manual Workflow User Guide (P/N 702990, ThermoFisher Scientific, Life Technologies, Carlsbad, CA 92008 USA). Briefly, 100 ng of total genomic DNA was denatured and then amplified. The amplified DNA was fragmented, precipitated and then centrifuged. The pellets were dried and resuspended in a buffer and added to a hybridization master mix, followed by hybridization to the microarray plate (Axiom $^{\text {Tw }}$ Equine Genotyping Array), in the GeneTitan ${ }^{\text {Tw }}$ Multi-Channel Instrument for $23.5 \mathrm{~h}$. Moreover, the array plate was labelled with biotin and FAM, washed and further stained with streptavidin and $\alpha F A M$ antibody. Finally, scanning was performed in the Gene $\operatorname{Titan}^{\mathrm{Tm}}$ Multi-Channel Instrument.

\section{CNV data analysis and QC}

Copy number calling was performed in the Axiom ${ }^{\mathrm{Tm}}$ Analysis Suite 1.1 according to the Best Practices Analysis Workflow described in Axiom ${ }^{\text {tw }}$ Genotyping Solution Data Analysis Guide (P/N 702961, ThermoFisher Scientific, Life Technologies, Carlsbad, CA 92008 USA) [43]. The threshold for the Dish QC quality metric was set to 0.82 according to the manufacturer's instructions. Following standard protocol, only the samples passing Call Rate $>96 \%$ were retained. The Axiom ${ }^{\circ}$ CNV summary software tool was used to generate input files for $\mathrm{CNV}$ prediction analysis, which were further processed using Nexus Copy Number ${ }^{\mathrm{mm}} 9.0$ (BioDiscovery, Hawthorne CA 90250, U.S.A.).

\section{Determination of population structure and group-specific CNV clusters}

Principal component analysis (PCA) of the SNP B-allelic frequency ratios of the samples and signal intensity of 
the copy number changes of the sample segments was done using the prcomp function in R v13.1 [44].

For the detection of breed specific SNP CNVs, we defined any SNP CNV identical between two or more horses across breeds as a shared SNP CNV. Similarly, for the detection of breed group-specific SNP CNVs, the eight breeds were grouped into three breed clusters according to the PCA analysis: Draught (Ardenner, Belgian draught, German draught, Exmoor ponies, Vlaams paard), Warmbloods (Belgian Warmblood and Swedish Warmblood), and Friesian horses. The Belgian draught horses showed the least variation in SNP CNVs and had a large sample size among the breeds studied. Therefore, the mean signal intensity of these individuals was used as a reference to discriminate between $\mathrm{CNV}$ gain (increased signal in relation to the average) and CNV loss (decreased signal in relation to the average).

The analysis of unique private SNP CNVs within breed groups was done using in house scripts, considering any SNP CNVs detected only in a single breed group (not shared with other breed groups) as a "unique private" breed group-specific SNP CNV.

\section{Determination of CNVR, gene annotation and PANTHER analysis}

First, those segment CNVs that overlapped by at least 1 bp were summarized as a single CNVR using the BEDTools software (-merge Bed command) [45]. Second, we determined the overlap (when at least $1 \mathrm{bp}$ was in common) between CNVRs identified in our study with the CNVRs already available online (DGVa, https://www.ebi. ac.uk/dgva) or published in recent scientific literature [20] on different population sizes and methods using BEDTools software (-intersect Bed command). This approach allowed us to identify CNVRs that had not been discovered so far (i.e. novel CNVRs) and CNVRs that are common between a wide range of equine breeds worldwide. The overlap between the CNVRs identified in the whole cohort of horses studied and genes annotated within the CNVRs in the horse genome (based on the EquCab2.0 sequence assembly), was determined using Variant Effect Predictor (VEP [46];) from the bioinformatics database Ensembl (http://www.ensembl.org/ ). Overrepresentation test was performed using the PANTHER (Protein ANalysis THrough Evolutionary Relationships, version 11.0; http://www.pantherdb.org/) classification system for the classification of genes by their molecular function, biological process, cellular component and protein class with default Bonferroni correction and false discovery rate (FDR) [47]. The identified CNVRs were also compared to previously reported trait QTLs in the horse (downloaded from the horse QTL database [48];) using bed file comparisons in BEDTools software (-merge Bed command) [45].

\section{Validation by RT-qPCR}

Validation of four genomic regions containing CNVRs was performed by real-time quantitative PCR (RT-qPCR) on the StepOne ${ }^{\mathrm{TM}}$ Real-Time PCR System (Applied Biosystems by Life Technologies, Darmstadt, Germany). These CNVRs were located within annotated genes. Primers and probes were designed using the Custom TaqMan ${ }^{\circledast}$ Assay Design Tool (Additional file 4: Table S3). Twenty horses were available for validation which belonged to three breeds: Exmoor ponies, Swedish Warmblood horses and Friesian horses. Ten out of the 20 horses were used in the CNVRs calling from the Axiom Analysis, thus allowing direct comparison of the results. DNA was prepared from 20 hair roots as described above (see Swedish Warmblood DNA Isolation protocol). DNA quality was determined with a Qubit ${ }^{\circ}$ Fluorometer. For each CNVR, 4 different concentrations were used to determine assay efficiency: $50 \mathrm{ng} / \mu \mathrm{L}, 25 \mathrm{ng} / \mu \mathrm{L}$, $12.5 \mathrm{ng} / \mu \mathrm{L}$ and $6.25 \mathrm{ng} / \mu \mathrm{L}$ of DNA. Reactions were assembled in a final volume of $15.00 \mu \mathrm{L}$, containing $1.50 \mu \mathrm{L}$ gDNA, $7.55 \mu \mathrm{L}$ PCR Master Mix 2X and $0.75 \mu \mathrm{L}$ of Custom TaqMan $^{\oplus}$ Assay mix $20 \mathrm{X}$ and $5.20 \mu \mathrm{L}$ of nuclease free water. The RT-qPCR conditions were as follows: initial denaturation at $95^{\circ} \mathrm{C}$ for $10 \mathrm{~min}$ (min), followed by 40 cycles of denaturation at $95^{\circ} \mathrm{C}$ for $15 \mathrm{~s}$ and combined annealing and extension at $60^{\circ} \mathrm{C}$ for $1 \mathrm{~min}$. Analysis was performed per each horse in duplicate and the average value was used for further calculations. The fold changes were determined using the $2^{-\Delta \Delta C t}$ method that normalises the $C_{t}$ values (cycle threshold) of the target gene with a reference gene $\left(\Delta C_{t}\right)$, and compare the $\Delta C_{t}$ to the $\Delta C_{t}$ of the reference sample [49]. We used as reference gene the Glyceraldehyde-3-phosphate dehydrogenase (GAPDH) gene on ECA6, according to previous CNVRs analyses in horses [18]. The reference individual was chosen among the 1755 horses and it displayed neither deletions nor duplications in the CNV prediction from the array in any of the four investigated CNVRs.

\section{Supplementary information}

Supplementary information accompanies this paper at https://doi.org/10. 1186/s12864-019-6141-z.

Additional file 1: Table S1. CNVRs identified in this study and including information regarding genome position, size, status, segment CNV counts, gene counts, number of individuals for each breed, QTL overlap and novelty.

Additional file 2. List of genes overlapping with the identified CNVRs. Additional file 3: Table S2. PANTHER gene ontology analysis of significantly over- and underrepresented genes.

Additional file 4: Table S3. Genomic regions validated for CNVs by RT-qPCR. Primer sequences, position, product size, annealing temperature and TaqMan probes are shown. The CNV status in the SNP-array and in the GPCR and the CNV validation by GPCR are given. Table S4. Results of quantitative PCR. The results of the four CNVs per each horse and the outcome of the validation through qPCR are presented. 


\section{Abbreviations}

CNV: Copy number variation; CNVR: Copy number variation region; ECA: Equus caballus autosome; FDR: False discovery rate; GAPDH: Glyceraldehyde-3-phosphate dehydrogenase; MNEC670K: Affymetrix Axiom $^{\text {TM }}$ Equine genotyping array; Olfr4F21: Olfactory receptor 4F21-like; OR10G2: Olfactory receptor 10G2; OR8S1: Olfactory receptor family 8 subfamily S member 1; PANTHER: Protein analysis through evolutionary relationships; PCA: Principal component analysis; RT-qPCR: Real-time quantitative polymerase chain reaction; SNP: Single nucleotide polymorphism; SV2C: Synaptic vesicle glycoprotein 2C; VEP: Variant effect predictor

\section{Acknowledgements}

We acknowledge the Exmoor Pony Society, Cullompton, United Kingdom, Swedish Warmblood Association, the Royal Friesian horse studbook, the Belgian Warmblood and the Belgian Draught horse association for providing samples.

\section{Authors' contributions}

$\mathrm{GL}, \mathrm{SM}, \mathrm{HK}, \mathrm{Al}, \mathrm{AB}$ designed the study. $\mathrm{AB}, \mathrm{NH}, \mathrm{HK}$ and $\mathrm{MS}$ performed CNV detection and the statistical analyses. MA and SM performed the RT-qPCR validation analysis. MS wrote the draft of manuscript. MS, MA, BV, NB, BJD, $L F, S J, A S, \AA V, S E, A l, S M, G L$ contributed to the concept of the study and improved the manuscript. All authors read and approved the final manuscript.

\section{Funding}

The research leading to these results has received funding from the European Union's. Seventh Framework Programme managed by REAResearch Executive Agency. http://ec.europa.eu/research/rea (FP7/2007-2013) under grant agreement number 606142. The Swedish-Norwegian Foundation for Equine Research funded genotyping of SWB horses, and part time salaries for SM, SE and $\AA \mathrm{V}(\mathrm{H} 1147215, \mathrm{SM})$. MS was supported by a grant from The Swedish Research council FORMAS to GL (2016-00947). The funding bodies played no role in the design of the study and collection, analysis, and interpretation of data and in writing the manuscript.

\section{Availability of data and materials}

The CNVRs identified in this study are available in Additional file 1. The datasets of this study are owned by different research groups or the partners of the EU HORSEGENE consortium (www.horsegene.eu or https://www.biw. kuleuven.be/GENLOG/horsegene/index.html). Data of the breeds Ardenner, Belgian draught horse, German draught, Vlaams paard and Belgian Warmblood are available upon reasonable request from the EU HORSEGENE project consortium, coordinator prof. Nadine Buys (nadine.buys@kuleuven. be), KU Leuven, Belgium. Data belonging to Exmoor ponies is available from the principal investigator Gabriella Lindgren (gabriella.lindgren@slu.se) or publicly available via Figshare (https://doi.org/10.6084/m9.figshare.3145759). The Swedish Warmblood dataset was generated and analysed in collaboration with the Swedish Warmblood Association and has a commercial value for them. The SWB horse data is therefore available from the principal investigator Sofia Mikko (sofia.mikko@slu.se) on reasonable request. Data belonging to Friesian horses is subject to owner consent and is available on reasonable request from the principal investigator Bart Ducro (bart.ducro@wur.nl).

\section{Ethics approval}

Hair samples from Friesian horses were collected with a written informed consent of the horse's owner. It was considered that there was no need for an Animal Care and Ethics Committee approval according to the Dutch law after consultation with the Animal Experiment Expert from Wageningen University \& Research. The Animal Welfare Officer thought it was not an animal experiment as referred to in the Dutch Act on Animal Experiments. Owners of the Exmoor ponies gave permission for their animals to be used in the study and the study was approved by the Ethics Committee for Animal Experiments in Uppsala, Sweden [Number: C 121/14 and number: 5.8.18-15453/2017]. Sample collection in Belgium was granted under P061/ 2012 and P096/2011, issued by the Animal Ethical Committee of KU Leuven. The hair samples for Swedish Warmbloods were collected prior to this study for pedigree verification purposes, with consent from the owners to use the samples also in research. The sample collection was in agreement with national legislation and did not require an ethical permit.

\section{Consent for publication}

Not applicable.

\section{Competing interests}

The authors declare that they have no competing interests.

\section{Author details}

${ }^{1}$ Department of Animal Breeding \& Genetics, Swedish University of Agricultural Sciences, Uppsala, Sweden. ${ }^{2}$ Department of Veterinary Science, Università di Parma, Parma, Italy. ${ }^{3}$ Department of Medical Sciences, Array and Analysis Facility, Uppsala University, Uppsala, Sweden. ${ }^{4}$ Faculty of Life and Environmental Science, University of Sydney, Sydney, NSW, Australia. ${ }^{5}$ Livestock Genetics, Department of Biosystems, KU Leuven, 3001 Leuven, Belgium. ${ }^{6}$ Animal Breeding and Genomics, Wageningen University \& Research, P.O. Box 338, 6700 AH Wageningen, the Netherlands. ${ }^{7}$ Centre for Genetic Resources, the Netherlands (CGN), Wageningen University \& Research, P.O. Box 338, 6700 AH Wageningen, the Netherlands.

Received: 29 May 2019 Accepted: 25 September 2019

Published online: 22 October 2019

\section{References}

1. Conrad DF, Pinto D, Redon R, Feuk L, Gokcumen O, Zhang Y, et al. Origins and functional impact of copy number variation in the human genome. Nature. 2010;464:704-12.

2. Mielczarek M, Fraszczak M, Nicolazzi E, Williams JL, Szyda J. Landscape of copy number variations in Bos taurus: individual - and inter-breed variability. BMC Genomics. 2018;19(1):410.

3. Saeed SS, Mohammadreza M, Dong-Dong W, Ali E. Detection of breedspecific copy number variations in domestic chicken genome. Genome. 2018;61:7-14

4. Serres-Armero A, Inna SP, Javier Q, Oscar R, Gabriel S, Lukas FKK, et al. Similar genomic proportions of copy number variation within gray wolves and modern dog breeds inferred from whole genome sequencing. BMC Genomics. 2017;18:997.

5. Ghosh S, Qu Z, Das PJ, Fang E, Juras R, Cothran EG, et al. Copy number variation in the horse genome. PLoS Genet. 2014;10:e1004712.

6. Weischenfeldt J, Symmons O, Spitz F, Korbel JO. Phenotypic impact of genomic structural variation: insights from and for human disease. Nature Rev Genet. 2013;14:125-38.

7. Doan R, Cohen N, Harrington J, Veazy K, Juras R, Cothran G, et al. Identification of copy number variants in horses. Genome Res. 2012;22: 899-907.

8. Chen C, Qiao R, Wei R, Guo Y, Ai H, Ma J, et al. A comprehensive survey of copy number variation in 18 diverse pig populations and identification of candidate copy number variable genes associated with complex traits. BMC Genomics. 2012;13:733.

9. Nicholas TJ, Baker C, Eichler EE, Akey JM. A high-resolution integrated map of copy number polymorphisms within and between breeds of the modern domesticated dog. BMC Genomics. 2011;12:1.

10. Maher B. Personal genomes: the case of the missing heritability. Nature. 2008:456:18-21.

11. Salmon Hillbertz NH, Isaksson M, Karlsson EK, Hellmén E, Pielberg GR, Savolainen P, et al. Duplication of FGF3, FGF4, FGF19 and ORAOV1 causes hair ridge and predisposition to dermoid sinus in ridgeback dogs. Nat Genet. 2007;39:1318-20.

12. Rosengren PG, Golovko A, Sundstrom E, Curik I, Lennartsson J, Druml T, et al. A cis-acting regulatory mutation causes premature hair graying and susceptibility to melanoma in the horse. Nat Genet. 2008:40:1004-9.

13. Rafati N, Andersson LS, Mikko S, Feng C, Raudsepp T, Pettersson J, et al. Large Deletions at the SHOX Locus in the Pseudoautosomal Region Are Associated with Skeletal Atavism in Shetland Ponies. G3 (Bethesda). 2016; 6(7):2213-23.

14. Demuth JP, Bie TD, Stajich JE, Cristianini N, Hahn MW. The evolution of mammalian gene families. PLoS One. 2006;1(1):e85.

15. Ghosh S, Das PJ, McQueen CM, Gerber V, Swiderski CE, Lavoie JP, et al. Analysis of genomic copy number variation in equine recurrent airway obstruction (heaves). Anim Genet. 2016;47:334-44.

16. Wang W, Wang S, Hou C, Xing Y, Cao J, Wu K, et al. Genome-wide detection of copy number variations among diverse horse breeds by array CGH. PLoS One. 2014;9:e86860. 
17. Doan R, Cohen ND, Sawyer J, Ghaffari N, Johnson CD, Dindot SV. Wholegenome sequencing and genetic variant analysis of a quarter horse mare. BMC Genomics. 2012;13:78.

18. Metzger J, Philipp U, Lopes MS, da Camara MA, Felicetti M, Silvestrelli M, et al. Analysis of copy number variants by three detection algorithms and their association with body size in horses. BMC Genomics. 2013;14:487.

19. Dupuis MC, Zhang Z, Durkin K, Charlier C, Lekeux P, Georges M. Detection of copy number variants in the horse genome and examination of their association with recurrent laryngeal neuropathy. Anim Genet. 2012;44(2): 206-8.

20. Schurink A, da Silva V, Velie B, Dibbits B, Crooijmans R, Francois L, et al. Copy number variations in Friesian horses and genetic risk factors for insect bite hypersensitivity. BMC Genet. 2018;19:1-13.

21. Xu L, Hou Y, Bickhart DM, Zhou Y, el HA H, et al. Population-genetic properties of differentiated copy number variations in cattle. Sci Rep. 2016;6:23161.

22. Paudel Y, Madsen O, Megens HJ, Frantz LAF, Bosse M, Crooijmans RPMA, et al. Copy number variation in the speciation of pigs: a possible prominent role for olfactory receptors. BMC Genomics. 2015;16(1):330.

23. Upadhyay M, da Silva VH, Megens HJ, Visker MHPW, Ajmone-Marsan P, Bâlteanu VA, et al. Distribution and functionality of copy number variation across European cattle populations. Front Genet. 2017;8:108.

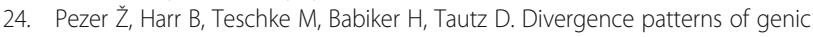
copy number variation in natural populations of the house mouse (Mus musculus domesticus) reveal three conserved genes with major populationspecific expansions. Genome Res. 2015;25(8):1114-24.

25. Schaefer RJ, Schubert M, Bailey E, Bannasch DL, Barrey E, Bar-Gal GK, et al. Developing a 670k genotyping array to tag 2M SNPs across 24 horse breeds. BMC Genomics. 2017:18:565.

26. Putnová L, Štohl R, Vrtková I. Genetic monitoring of horses in the Czech Republic: a large-scale study with a focus on the Czech autochthonous breeds. J Anim Breed Genet. 2018;135(1):73-83.

27. Petersen JL, Mickelson JR, Rendahl AK, Valberg SJ, Andersson LS, Axelsson J, et al. Genome-wide analysis reveals selection for important traits in domestic horse breeds. PLoS Genet. 2013;9(1):e1003211.

28. Librado P, Fages A, Gaunitz C, Leonardi M, Wagner S, Khan N, et al. The evolutionary origin and genetic makeup of domestic horses. Genetics. 2016; 204(2):423-34

29. Andersson LS, Larhammar M, Memic F, Wootz H, Schwochow D, Rubin CJ, et al. Mutations in DMRT3 affect locomotion in horses and spinal circuit function in mice. Nature. 2012;488:642-6.

30. Imsland F, McGowan K, Rubin CJ, Henegar C, Sundström E, Berglund J, et al. Regulatory mutations in TBX3 disrupt asymmetric hair pigmentation that underlies dun camouflage color in horses. Nature Genet. 2016;481:52-158.

31. Leroy G, Callède L, Verrier E, Mériaux JC, Ricard A, Danchin-Burge C, et al. Genetic diversity of a large set of horse breeds raised in France assessed by microsatellite polymorphism. Genet Sel Evol. 2009;41:5.

32. Molin A, Berglund J, Webster MT, Lindblad-Toh K. Genome-wide copy number variant discovery in dogs using the CanineHD genotyping array. BMC Genomics. 2014;15:210.

33. Yang L, Xu L, Zhou Y, Liu M, Wang L, Kijas JW, et al. Diversity of copy number variation in a worldwide population of sheep. Genomics. 2018; 110(3):143-8.

34. Skujina I, Winton CL, Hegarty MJ, McMahon R, Nash DM, Davies Morel MCG, et al. Detecting genetic regions associated with height in the native ponies of the British Isles by using high density SNP genotyping. Genome. 2018; 61(10):767-70.

35. Gottschalk M, Metzger J, Martinsson G, Sieme H, Distl O. Genome-wide association study for semen quality traits in German Warmblood stallions. Anim Reprod Sci. 2016:171:81-6.

36. Cook D, Gallagher PC, Bailey E. Genetics of swayback in American Saddlebred horses. Anim Genet. 2010;41(Suppl. 2):64-71.

37. Haase B, Signer-Hasler H, Binns MM, Obexer-Ruff G, Hauswirth R, Bellone RR, et al. Accumulating mutations in series of haplotypes at the KIT and MITF loci are major determinants of white markings in Franches-Montagnes horses. PLoS One. 2013;8(9):e75071.

38. Chain FJJ, Feulner PGD, Panchal M, Eizaguirre C, Samonte IE, Kalbe M, et al. Extensive copy-number variation of young genes across stickleback populations. PLoS Genet. 2014;10(12):e1004830.

39. Bekaert M, Conant GC. Gene Duplication and Phenotypic Changes in the Evolution of Mammalian Metabolic Networks. Jordan IK, ed. PLoS One. 2014; 9(1):e87115.
40. Sun BB, Maranville JC, Peters JE, Stacey D, Staley JR, Blackshaw J, et al. Genomic atlas of the human plasma proteome. Nature. 2018;558(7708):73-9.

41. Redon R, Ishikawa S, Fitch KR, Feuk L, Perry GH, Andrews TD, et al. Global variation in copy number in the human genome. Nature. 2006;444(7118): 444-54.

42. Velie BD, Shrestha M, François L, Schurink A, Tesfayonas YG, Stinckens A, et al. Using an inbred horse breed in a high density genome-wide scan for genetic risk factors of insect bite hypersensitivity (IBH). PLoS One. 2016;11(4): e0152966.

43. Axiom Genotyping Solution Data Analysis Guide (P/N 702961; http://www. bea.ki.se/documents/axiom_genotyping_solution_analysis_guide.pdf). Accessed May 2018.

44. R Development Core Team. R: A Language and Environment for Statistical Computing. Vienna: R Foundation for Statistical Computing; 2011.

45. Quinlan AR, Hall IM. BEDTools: a flexible suite of utilities for comparing genomic features. Bioinformatics. 2010;26:841-2.

46. McLaren W, Gil L, Hunt SE, Riat HS, Ritchie GR, Thormann A, et al. The Ensembl variant effect predictor. Genome Biol. 2016;17(1):122.

47. Mi H, Huang X, Muruganujan A, Tang H, Mills C, Kang D, et al. PANTHER version 11: expanded annotation data from gene ontology and Reactome pathways, and data analysis tool enhancments. Nucleic Acids Res. 2017; 45(D1):D183-9.

48. Hu Z, Park CA, Reecy JM. Developmental progress and current status of the animal QTLdb. Nucleic Acids Res. 2016:44:D827-33.

49. Livak KJ, Schmittgen TD. Analysis of relative gene expression data using realtime quantitative $P C R$ and the $2-\Delta \Delta C T$ method. Methods. 2001;25:402-8.

\section{Publisher's Note}

Springer Nature remains neutral with regard to jurisdictional claims in published maps and institutional affiliations.

Ready to submit your research? Choose BMC and benefit from

- fast, convenient online submission

- thorough peer review by experienced researchers in your field

- rapid publication on acceptance

- support for research data, including large and complex data types

- gold Open Access which fosters wider collaboration and increased citations

- maximum visibility for your research: over $100 \mathrm{M}$ website views per year

At BMC, research is always in progress.

Learn more biomedcentral.com/submissions 